\title{
Lumen
}

Selected Proceedings from the Canadian Society for Eighteenth-Century Studies

\section{Les almanachs d'amour au siècle des Lumières}

\section{Stéphanie Loubère}

Volume 28, 2009

URI : https://id.erudit.org/iderudit/1012038ar

DOI : https://doi.org/10.7202/1012038ar

Aller au sommaire du numéro

Éditeur(s)

Canadian Society for Eighteenth-Century Studies / Société canadienne d'étude du dix-huitième siècle

ISSN

1209-3696 (imprimé)

1927-8284 (numérique)

Découvrir la revue

Citer cet article

Loubère, S. (2009). Les almanachs d'amour au siècle des Lumières. Lumen, 28, 69-81. https://doi.org/10.7202/1012038ar

Copyright (c) Canadian Society for Eighteenth-Century Studies / Sociéte canadienne d'étude du dix-huitième siècle, 2009
Ce document est protégé par la loi sur le droit d'auteur. L'utilisation des services d'Érudit (y compris la reproduction) est assujettie à sa politique d'utilisation que vous pouvez consulter en ligne.

https://apropos.erudit.org/fr/usagers/politique-dutilisation/ 


\section{4 : Les almanachs d'amour au siècle des Lumières}

Après la littérature religieuse, les almanachs occupent la seconde place dans la production littéraire populaire du siècle des Lumières. Ils proposent de façon concise et "portative» des renseignements pratiques comme le calendrier de l'année en cours et les dates relatives à des événements astronomiques (éclipses, lunaisons, etc.), religieux (fêtes, jours fériés rassemblés dans le comput, avec le nombre d'or, le cycle solaire, l'indiction romaine) ou sociaux (foires notamment). Ils rassemblent également des conseils techniques relatifs aux travaux agricoles : le Grand Compost des bergers ou L'Almanach du bon laboureur furent de grands succès éditoriaux. À partir de la deuxième moitié du XVIII siècle, ils s'enrichissent $\mathrm{d}^{\prime}$ anecdotes, et de textes courts et divertissants (fables, contes, chansons, etc.).

Originellement destinés à un public peu ou pas lettré (il n'est pas réellement besoin de savoir lire pour les consulter), les almanachs s'insinuent progressivement dans tous les milieux et se mettent au service de discours très différents, prenant des formes «non populaires» : l'almanach devient une mode, au point que Nicolas Le Camus de Mézières lui consacre une "analyse critique et raisonnée» dans un essai intitulé L'Esprit des almanachs (Paris, veuve Duchesne, 1783).

L'almanach d'amour constitue l'un des avatars les plus étonnants de cette production florissante, et peut nous renseigner sur les mutations érotiques et érotographiques du siècle.

Les almanachs d'amour détournent un média à l'origine populaire pour en faire un objet littéraire à l'usage de l'élite libertine. Leur saveur et leur valeur résident donc dans le rapport parodique qu'ils entretiennent avec les almanachs traditionnels. Matériellement, ces almanachs

1 Voir la «Petite histoire de l'almanach» dans l'ouvrage de Geneviève Bollème, Les Almanachs populaires aux XVIr et XVIII siècles. Essai d'histoire sociale, Paris, La Haye, Mouton et Cie, 1969. 
ressemblent à leurs confrères roturiers : même type de format (portatif), incluant un calendrier, éventuellement des pages blanches (un agenda), et un espace réservé pour les «comptes» — non pas du ménage, on s'en doute, mais du jeu (avec des pages pour les pertes et les gains). Le reste est occupé par des variantes parodiques du comput ecclésiastique, de l'annonce des fêtes et foires, des conseils aux jardiniers ou encore des prédictions, le tout assorti de poèmes et chansons de thématique érotique (dans la veine des Étrennes, forme également florissante à partir du milieu du siècle).

On peut distinguer différentes catégories dans le corpus très vaste des almanachs d'amour: almanachs oraculaires, almanachs allégoriques, almanachs poissards et pornographiques, almanachs bijoux. Tous ont en commun de détourner les fonctions originelles de l'almanach traditionnel. Les «almanachs oraculaires» imitent une forme très répandue d'almanachs et sont une variante de la tradition médiévale des questions d'amour. Ils prétendent donner des prédictions, au moyen de tables d'une utilisation parfois complexe. La table de prédiction de l'Almanach de l'amour et de la fortune (1779) permet ainsi au lecteur, par une série de calculs, de trouver «les réponses les plus divertissantes» aux soixante questions qu'il peut se poser, et de savoir par exemple «Si [s]a maîtresse [l]'aime mieux que [s]on rival», «Si l'on sera bientôt marié», etc.

Les «almanachs allégoriques» parviennent à établir un pont entre la tradition de la cartographie tendre et celle des éphémérides dans les almanachs. Ils privilégient la description non pas de l'espace amoureux, mais de sa temporalité, et complètent les descriptions de voyage dans les contrées allégoriques. Contemporaine de la Clélie, une petite vogue d'almanachs allégoriques avait déjà exploité cette veine: le Grand Almanach d'amour (1657) de Samuel Isarn contient la description d'un «Voyage de la province d'amour» qui répond à la carte de Tendre proposée par Mlle de Scudéry, mais le modèle allégorique le plus complet est sans doute l'Almanach d'amour paru en 1668. Presque tous les almanachs allégoriques du XVIII siècle l'imiteront ou lui emprunteront une partie de son contenu. On y trouve les «douze signes de l'amour» (les soupirs, la pâleur, le respect, la ruserie, la prodigalité, etc.) et les «mois de l'année d'amour» (visite, complaisance, déclaration, possession, soupçon, jalousie, etc.), qui sont une transposition temporelle du modèle cartographique de Tendre. L'auteur consacre également une page à chaque mois de l'année et à ses saints, ainsi qu'aux lunaisons (les quartiers de lunes sont dessinés en forme de cœur plus ou moins plein : Isarn avait inauguré ce joli subterfuge). Viennent ensuite la «Prédiction générale pour l'année», les «Éclipses d'amour» («la canicule d'amour [...] commencera le 22 de Possession jusqu'au 22 d'Attachement»), la 
«Prédiction sur les quatre saisons de l'année», l'«Équinoxe», «Les foires du Royaume d'Amour», et enfin «Le moyen très nécessaire et très utile pour cueillir les fruits de l'amour». Cette parodie très complète du Compost des bergers nourrit fort bien l'imaginaire précieux friand de «bergeries» en tous genres.

D'autres textes assument la fonction descriptive des almanachs de façon réaliste et non plus allégorique, et se livrent à un détournement licencieux du modèle populaire. Certains almanachs au titre toujours suggestif (Almanach polisson, Étrennes poissardes et polissonnes, Le Gaillard de bonne humeur, etc.) ont pour seul but de proposer un divertissement égrillard. Si l'on considère qu'ils s'adressent à la frange supérieure de la société, ils témoignent du goût pour un certain exotisme social, en s'intéressant au comportement amoureux des petites gens sous le prétexte que «le villageois aime ainsi que les rois» (Almanach poissard, 1757). Certains de ces almanachs ont des titres «sages», mais leur contenu l'est en revanche beaucoup moins : les Étrennes libertines, au frontispice obscène (1743), les scatologiques Étrennes du sentiment (sous titrées sans vergogne: Almanach chantant de la sagesse, 1770), les Étrennes aux amateurs de Vénus (1787), recueil de chansons dont le sens impudique n'apparaît qu'à la lumière des gravures qui leur sont apposées, ou le plus trompeur de tous, l'Almanach des honnêtes femmes de Maréchal (1790), recèlent de joyeuses obscénités. D'autres almanachs licencieux sont plus directs, tels l'Almanach de Priape (1741) ou les Étrennes aux fouteurs (1793).

Les "almanachs bijoux" sont l'exemple extrême du désistement utilitaire des almanachs d'amour. Petites choses extrêmement délicates et luxueuses, ils tiennent à peine du livre : richement ornés, le Bijou mignon des dames (1769), L'Almanach couleur de rose (1771) ou les Étrennes galantes (1778) peuvent être portés en pendentifs'; ce sont des objets plus faits pour être exhibés que consultés. Dénué de toute fonction pratique, l'almanach breloque est un objet paradoxal : inutilisable, il s'affirme comme emblème luxueux d'une société oisive.

Ce rapide survol nous permet de constater que les almanachs d'amour couvrent tout le champ de l'imaginaire érotique, et proposent une vision de l'amour qui va de la plus éthérée (almanachs allégoriques) à la plus réaliste (almanachs poissards et licencieux). Le jeu parodique avec la forme populaire de l'almanach n'a pas produit de chef d'œuvre, mais son insistance nous renseigne sur l'effervescence créative de l'érotographie des Lumières. L'almanach d'amour se présente comme

2 Les Étrennes galantes sont vendues « chez Vallages, marchand bijoutier ». 
une version portative de la science amoureuse, un compendium qui reprend sous forme brève les préceptes diffusés de façon extensive dans les romans ou dans les arts d'aimer qui fleurissent à l'époque. Dans les chansons et les parodies faciles des almanachs, on trouve comme un précipité de l'érotique diffusée par les romans, et leur aspect ludique cache peut-être des enjeux profonds : nous pouvons y suivre les évolutions de l'imaginaire qu'ils véhiculent et le portrait en ombre chinoise du public auquel ils s'adressent et dont ils remplissent les attentes. À travers eux se dit le fantasme de constituer une science de l'amour ainsi que l'obsession libertine du temps.

L'almanach d'amour se présente souvent comme un mémento de ruses, un vade-mecum à l'usage des petits maîtres. Il affiche donc une ambition didactique : donner les rudiments d'un art d'aimer ou de séduire. Ce type d'almanach imite un discours qui propage un savoir technique. Cela l'oblige d'une façon ou d'une autre à soutenir ou à mettre en question l'assimilation de l'amour à un savoir-faire qui résulte de l'utilisation allégorique ou parodique de la forme almanach.

Il se présente ironiquement comme un outil de "vulgarisation», alors que la leçon de séduction libertine se doit d'être élitaire, et fonctionne bien souvent comme une initiation: l'almanach d'amour, qui utilise une forme "publique» pour diffuser un savoir secret, est à l'opposé de la leçon que le Versac de Crébillon délivre à Meilcour dans une «promenade solitaire ${ }^{3}$, à l'abri des oreilles indiscrètes.

La forme brève de l'almanach impose l'idée d'un savoir érotique borné, réduit à quelques principes qu'il suffit d'appliquer. L'almanach d'amour donne forme au fantasme de la leçon de séduction, telle qu'elle se présente par exemple dans les romans libertins : l'Almanach perpétuel d'amour de 1681 affiche déjà son ambition de "renferme[r] tout ce qui est plus important pour la conduite ordinaire des amants, et [d']apprend[re] ce qui vient le plus souvent en pratique». Approuvé par les «astrologues cupidoniciens» et un «professeur ès mathématiques d'amour", il impose une vision proprement pragmatique de l'amour.

Cette assimilation parodique de l'amour à un savoir empirique, élaboré dans l'observation objective des phénomènes, traduit l'émergence d'une nouvelle conception de l'amour, et d'une volonté d'étendre le champ d'action de la raison à la sphère des sentiments et des sensations. L'almanach d'amour soumet ainsi l'amour à l'esprit positif, et le fait redescendre des sphères éthérées où il est longtemps resté confiné. L'auteur de l'Almanach terrestre et facétieux (1717) veut

3 Crébillon, Les Égarements du car et de l'esprit, Paris, Garnier, 2000, p. 208. 
ainsi faire un almanach «en rampant», en observant non les astres mais ce qui se passe sur terre :

Mon observatoire, c'est mon cabinet. [...] Je n'ai qu'à contempler la terre, me ressouvenir de ce qui s'y fait à présent, et prévoir ce qui s'y fera dans la suite.

Il prétend faire des prédictions non en vertu d'un don magique, mais en déduisant des lois rationnelles de ses observations : la raison se substitue à la superstition et fait de l'almanach un objet des Lumières. Cet Almanach terrestre, comme les almanachs populaires, fait appel au «bon sens» de ses lecteurs, et bien souvent à leur esprit critique. Il n'est pas le seul : même un Almanach des Grâces (1788), chansonnier sans prétention, se signale par un tel appel à l'esprit critique de son lecteur, à travers le diagnostic d'une amante qui décrypte froidement le comportement de son amant, par observation et déduction :

Quand sous ses lois l'amour nous range

On ne dort, ne boit, ni ne mange ;

Toi tu fais tes quatre repas :

Non, mon ami, tu n'aimes pas.

L'almanach invite à interpréter les signes, à déduire la nature de l'amour de l'observation de symptômes physiques (voir déjà le Traité des passions de Descartes). Sous l'allure légère de semblables chansons, on trouve affirmée une solide défense du discernement et du bon sens éclairé par la science

Si la Carte de Tendre proposait une allégorie spatiale des progrès de l'amour, l'almanach d'amour se présente comme une allégorie temporelle, qui s'accorde avec l'obsession libertine du temps.

La description d'une temporalité cyclique (retour des saisons) qui est généralement celle de l'almanach coïncide avec la temporalité elle aussi cyclique du manège libertin. La ponctuation du calendrier du jardinier insiste sur les périodes propices à telle ou telle intervention de l'homme sur la nature ; le calendrier amoureux adopte une pratique comparable. Le moment, cette notion si importante dans la séduction libertine des Lumières, trouve dans l'almanach un support inégalable. L'obsession du temps propice s'y déploie notamment à travers la recherche de $l^{\prime}$ heure du berger - avec un retour à la source de la métaphore ${ }^{4}$. L'avis

4 L'étoile du berger n'est autre que Vénus : premier astre du soir, elle signalait aux bergers le moment de rassembler leurs troupeaux pour les mettre à l'abri. 
au lecteur de l'Almanach galant (1741) prévient ainsi que «ce précieux moment doit être ménagé et saisi à propos, car quand il est une fois perdu, il ne se peut retrouver».

L'almanach d'amour s'inscrit également dans l'engouement pour toute la littérature éphémère : c'est un objet dont la durée de vie est limitée, à l'image des bulles de savon que s'amusent à faire les chérubins ailés sur le frontispice de l'Almanach mignon (1762). Le plaisir que procure sa lecture est lui-même voué à être provisoire, sa caducité est peut-être ce qui fait son charme, car elle s'accorde avec les leçons de carpe diem que bien souvent il véhicule. Amour volage et littérature éphémère se rejoignent dans la figure de la «femme almanach» que propose le Gaillard de bonne humeur, «almanach chantant et divertissant» de 1786, où un mari répond à sa femme qui veut être livre pour être plus souvent avec lui : «Soyez livre, je le veux bien, pourvu que vous soyez un almanach, afin que je puisse en changer tous les ans».

Enfin, les almanachs d'amour participent à l'actualisation du débat amoureux, et proposent une chronique discontinue mais fidèle du libertinage. Les almanachs d'amour mesurent le temps, et construisent une temporalité : leur chronométrie est également une chronologie. L'ordre dans lequel ils présentent les saisons est révélateur du type d'idéologie érotique qui les sous-tend. La plupart suivent l'ordre des saisons du printemps à l'hiver, à l'instar des almanachs populaires, et les écarts par rapport à ce modèle sont toujours porteurs de sens. Ainsi, la séquence hiver - printemps - été - automne suggère une temporalité où le temps de l'épanouissement et de la jouissance est encadré par les saisons de la stérilité et de la dégradation. Métaphoriquement, l'amour est menacé par la vieillesse et la mort, l'entreprise amoureuse est présentée comme vouée à une déliquescence irrémissible. C'est la séquence choisie par Isarn pour son Grand Almanach, dans la logique de sa vision d'un Tendre soumis à la circularité et à la déréliction ${ }^{5}$. La séquence printemps - été - automne - hiver, au contraire, présente un cycle reposant sur le principe de renouvellement, ou laissant du moins possible une lecture régénératrice et positive de la succession des saisons. C'est ainsi qu'en 1741, l'Almanach galant dédié au beau sexe, qui s'inspire de très près de l'Almanach d'amour de 1668 , opère sur le plan chronologique des modifications révélatrices. Après les mois de la séduction, on trouve les mois de «Tendresse assurée» (au lieu de Pos-

5 On se souvient que la carte de Tendre est bordée à l'Est par le Lac d'Indifférence, à l'Ouest par la Mer d'Inimitié et au Nord par la Mer Dangereuse et les Terres Inconnues. 
session), d' «Engagement Solide» (au lieu de «Soupçon») et les derniers mois sont «Mariage Heureux, Contentement parfait, Plaisirs sans Fin». De façon flagrante, le cycle de progrès et de chute est remplacé par la description d'une ascension continue vers un acmé (le mariage), évacuant de ce fait à la fois l'inquiétude qui habite l'érotique Tendre et les cycles désinvoltes du libertinage. On peut trouver, outre ces différences $\mathrm{d}^{\prime}$ ordre chronologique qui manifestent subjectivement une vision positive ou négative de la temporalité amoureuse, des agencements qui échappent à la chronologie régulière. En insufflant le désordre là où le plus d'ordre est attendu - quoi de plus immuable que le retour des saisons? - l'almanach est investi d'une énergie particulière, devient une force anarchique - ou du moins rend compte du pouvoir anarchique de l'amour. L'Almanach terrestre de 1717 en est un exemple : il brouille les séquences et présente les saisons dans le désordre (printemps, automne, hiver, été).

Le rôle métaphorique des saisons de l'amour est ainsi très important : l'amour présenté comme un cycle sans cesse renouvelé suggère inévitablement un type d'érotique libertine marqué par l'inconstance et la répétition, alors que les almanachs qui insistent sur son développement invitent à penser les progrès ou la décadence de l'amour, et donc à opter pour une érotique qui prône soit la fidélité soit l'hédonisme du carpe diem. Le jeu plaisant qu'autorise le découpage du temps en périodes érotiques peut avoir des implications beaucoup plus profondes. L'agencement minutieux du temps social a longtemps été l'un des plus tenaces adjuvants de l'emprise religieuse. Selon Jean-Louis Flandrin, la codification temporelle de la sexualité et de la vie quotidienne en général obéit à l'appropriation du temps social par la christianisation, qui a substitué un calendrier chrétien aux calendriers païens. Dans Un temps pour embrasser. Aux origines de la morale sexuelle occidentale, il cite l'Ecclésiaste (III, 2-8), et ses prescriptions :

Il est un temps pour chaque chose.

Un temps pour aimer et un temps pour hair.

Un temps pour embrasser et un temps pour fuir les embrassements.

Les pénitentiels chrétiens, édictés pendant plusieurs siècles par les autorités ecclésiastiques, sont intervenus dans la vie amoureuse des fidèles, en autorisant notamment les relations sexuelles selon des règles

6 Jean-Louis Flandrin, Un temps pour embrasser. Aux origines de la morale sexuelle occidentale, Paris, Seuil, 1983, p. 5. 
draconiennes. Après considération et calcul de tous les interdits relatifs aux menstruations, aux grossesses, aux fêtes religieuses, aux carêmes, etc., il restait environ un jour sur quatre aux époux pour accomplir leur devoir conjugal ${ }^{7}$. L'air de rien, les almanachs d'amour opposent à cette arithmétique drastique du calendrier religieux un découpage du temps tout entier voué à l'amour, sans interdits ni restrictions. À leur échelle discrète, les almanachs d'amour ont ainsi pu mener leur combat contre l'Infâme, surtout lorsque, reliés ainsi que des missels, les galants les ouvraient pendant l'office, et, comme le rapporte John Grand-Carteret, «tout en remplissant [leurs] devoirs religieux, pouvai[ent], à part soi, dire la messe de Cythère ${ }^{8}$ ».

Un autre rôle s'offrait aux almanachs d'amour : dans les almanachs licencieux qui se diffusent vers la fin du siècle, on assiste à la diffusion graduelle des «dangereux secrets» et à la banalisation de l'acte amoureux, abordé de façon pragmatique et non plus morale. L'almanach avait ici un rôle à jouer : ouvrage empruntant une démarche didactique prétendument scientifique, il n'a pas besoin de se justifier outre mesure pour aborder la chose amoureuse sous son aspect le plus cru. L'almanach ne se préoccupe pas d'institutionnaliser le discours physiologique sur l'amour, il est rarement polémique ou combatif, mais en intégrant naturellement ce discours il contribue à l'effort typologique et terminologique mené par les théoriciens libertins. Il y a dans l'almanach comme une facilité à nommer et classer ce qui est si longtemps resté «innommable». Très tôt, la couverture parodique et scientifique de l'almanach permet ce que Chantal Thomas qualifie de jeu «entre l'innommable du sexe et l'explicite du texte ${ }^{9}$ ». C'est ainsi que l'on peut trouver dans un almanach précoce et plutôt précieux, des incartades «techniques» étonnantes : l'Almanach perpétuel de 1681 parsème ses descriptions des diverses heures du cadran de Tendre par des remarques et précisions sans façon. Le retour en vigueur qui survient lors de la troisième heure nocturne, après les premiers ébats, se perçoit par «Le signum salutis, vous m'entendez bien; c'est-à-dire quand Virga Aurea lève la tête». On y trouve aussi un chapitre explicatif

7 Ibid., p. 54 .

8 John Grand-Carteret, Les Almanachs français, 1600-1895, Paris, Alisie et C $C^{\mathrm{ie}}, 1896$, p. xxxvii.

9 À propos des pamphlets révolutionnaires en particulier et de la littérature licencieuse en général, dans son article «La foutromanie révolutionnaire», dans L'Enfer de la Bibliothèque Nationale, tome VI, Paris, Fayard, 1987, p. 267-279. 
sur la "petite oie», que les précepteurs de Thérèse philosophe, quelques trois quarts de siècle plus tard, ne renieraient pas totalement :

Ceux qui ont passé les heures du jour de la boussole diaire de Cupidon savent bien ce que c'est que petite oie. Toutefois, comme il y a des apprentifs en amour, qui ignorent la signification de ce terme, je leur dirai que ce n'est autre chose que les enjouements d'amour, savoir petits baisers, vues agréables, attouchements charmants et petites folâtreries : car comme les ragoûts que l'on fait des pieds, des ailes, de la tête d'une oie sont des invite-appétit pour manger le corps, de même Cupidon veut que les enfants s'animent à ses combats par ces petites allumettes d'amour.

Notons que l'auteur de cet almanach n'est pas aussi prolixe quand il s'agit de décrire les plaisirs de l'heure du berger ou de la jouissance. Il obéit néanmoins au même désir de donner le mot juste qui animera plus tard les Étrennes aux fouteurs (1793): «la crudité des expressions n'a rien de révoltant $[. .$.$] ce n'est plus l'homme qu'on entend, c'est la$ nature même».

L'almanach permet ainsi de faire passer le savoir érotique du secret à l'exhibition et permet aussi une approche systématique. L'almanach se prête bien à la liste, et permet de voir comment cette tentation toujours présente dans l'imaginaire amoureux se modifie au fil du temps : on passe de la liste des lieux de Tendre à celle des heures du Cadran de Vénus, pour arriver à la liste de l'Almanach des adresses des demoiselles de Paris (1791), qui se décrit comme un véritable "sérail portatif ${ }^{10}$ » et recense toutes les «prêtresses de Vénus» de la capitale, en donnant «leurs noms, demeures, âges, portraits, caractères, talents et le prix de leurs charmes", ou encore à la liste allègrement blasphématoire des nouvelles saintes du calendrier républicain, dans l'Almanach des honnêtes femmes pour l'année 1790 de Sylvain Maréchal. Maréchal illustre dans son almanach ce penchant pour le dénombrement et la typologie méthodique en matière érotique : il compose un calendrier où chaque mois est voué à un type particulier de volupté (celle pratiquée par les «fricatrices», les «tractatrices», les "fellatrices», les "phicidisseuses», les «chalcidisseuses», etc. ${ }^{11}$ ). Les fêtes chrétiennes sont méticuleuse-

10 «C'est un véritable sérail portatif. Pour vingt-quatre sols, un simple citoyen devient un véritable sultan, sans avoir les embarras du ménage.»

11 Cette terminologie est empruntée à l'ouvrage de Mirabeau, Erotika Biblion, paru en 1783. L'almanach de Maréchal est un exemple du transfert du discours érotique pratiqué par les auteurs libertins, dont il propose comme un précipité. 
ment remplacées par des fêtes bien charnelles. La Présentation devient la «Fête du bidet» («Beaucoup de femmes appellent un bidet leur confesseur : il efface tous les péchés par une ablution parfaite») ; la «Fête des maquereaux» se substitue à l'Annonciation («Ce changement ne fait aucun tort à notre Sainte Religion : tout le monde sait que le beau Gabriel était l'agent des plaisirs du Saint Esprit») ; et le Vendredi Saint se mue spirituellement en «Fête de la petite mort» («Pourquoi ce vendredi nous rappellerait-il la fin tragique d'un dieu, pendu il y a 1790 ans ? Faisons donc disparaître la sottise de nos pères, et remplaçons le Christ par cette mort délicieuse où nous plonge le plaisir. [...] Heureux si, comme le Sauveur du monde, nous pouvions rester trois jours de suite dans le tombeau, et en sortir encore triomphant»), etc. La propagation du savoir érotique va de pair avec celle de l'esprit philosophique : il n'est pas étonnant que l'almanach ait été par la suite l'une des formes de prédilection de la littérature pamphlétaire de la Révolution.

Produits éphémères de leur temps, la succession hétéroclite des almanachs d'amour rend compte de l'évolution des mentalités, et dessine une histoire de l'éros des Lumières. Les almanachs d'amour participent au débat érotique de leur temps, aussi bien pour laisser entendre les voix de la libération des mœurs que pour renforcer celles de la réaction. Il semble que la morale soit l'une des composantes les plus constantes de l'almanach : morale chrétienne, morale civile ou citoyenne (pour les almanachs révolutionnaires notamment), morale hédoniste, on y trouve à peu près tous les registres. L'Almanach perpétuel de 1717 propose ainsi des "Maximes de sagesse d'amour», et des "Règles de santé d'amour», dont les termes ne diffèrent guère de ceux que l'on pourrait trouver dans les préceptes de bonne morale transmis dans les almanachs traditionnels («Persévère à bien faire et espère toujours de mieux en mieux», "Contente-toi de ton sort et estime-toi heureux de ta fortune», "Aime l'honneur et la vertu si tu veux vivre heureux et content à jamais en amour», etc.). Même dans les ouvrages les plus subversifs, venus plus tard, on trouve toujours trace d'une visée moralisante, y compris lorsque la morale s'est laïcisée et qu'elle est devenue une morale citoyenne ou biologique : il y a toujours un «ordre» qu'il faut respecter (social, physiologique, ou autre). Les Étrennes aux fouteurs (1793) s'achèvent ainsi sur un "Avis aux lecteurs, et aux lectrices, car [il] en [aura] peut-être», qui tranche avec le reste de l'ouvrage, assez peu respectueux des «bonnes mœurs». L'auteur y enjoint pourtant plaisamment ses lecteurs de se consacrer aux plaisirs («Du seul plaisir éprouvez le souci»), tout en restant d'honnêtes citoyens («Mais que l'ordre et l'honneur soient toujours respectés») : n'est-ce pas la position adoptée par les plus extrémistes des libertins sadiens? 
Sous les abords inoffensifs de leur forme parodique, les almanachs $\mathrm{d}^{\prime}$ amour s'imposent discrètement mais efficacement comme un formidable outil de propagation idéologique. À leur futile mais insistante façon, ils témoignent de la remarquable force de subversion sociale, morale et littéraire du discours érotique des Lumières.

STÉPHANIE LOUBÈRE

Université Paris IV - Sorbonne

\section{Éléments Bibliographiques}

L'abondante production d'almanachs d'amour est essentiellement anonyme. Voici quelques uns des titres les plus significatifs, qui témoignent de l'extraordinaire fécondité de cette veine littéraire.

\section{Almanachs oraculaires}

Samuel Isarn, Grand Almanach d'amour, où sont contenues les prédictions générales de l'année et de chaque saison en particulier, avec un moyen très nécessaire pour savoir en quels temps et lieux il faut semer et cultiver toutes choses qui servent en amitié et en amour, Paris, Sercy, 1657.

Almanach terrestre et facétieux, ou l'Oracle enjoué, contenant des prédictions criti-comiques, satiriques et réjouissantes, qui s'accompliront pendant le cours de l'année 1717, Paris, Prault, au Paradis, 1717.

L'Oracle de Cythère, ou l'Almanach du berger, Paris, Duchêne, 1755.

Almanach du sort, ou Recueil de nouveaux oracles, Paris, Duchêne, 1757.

Les Tablettes de l'amour ou le nouvel oracle de Cythère, dans lequel on peut trouver son horoscope sur des airs connus. Ouvrage très agréable aux personnes heureuses et orné d'une préface intéressante pour l'acheteur, par M. $D^{* * *}$, Paris, Duchêne, 1760.

Almanach de l'amour et de la fortune. Les curieux trouveront les réponses les plus divertissantes aux soixante questions énoncées dans la table ci-après, Lille, Bloquel, 1779.

Almanach de l'amour et de la fortune, pour l'année 1779, contenant diverses réponses aux demandes que pourraient faire les personnes curieuses de connaitre l'avenir, à Delphes, par ordre du Destin, 1779.

Le Petit Devin de Cythère ou le plaisir des Belles, Paris, Cailleau, 1782.

Le Chef d'œuvre de l'amour, almanach enchanteur, Paris et Paphos, Bailly, 1786.

Les Prophéties galantes, ou le Palais du sort et de l'amour, Paris, Laurens, 1793. 


\section{Stéphanie Loubère}

\section{Almanachs allégoriques}

Samuel Isarn, Grand Almanach d'amour, où sont contenues les prédictions générales de l'année et de chaque saison en particulier, avec un moyen très nécessaire pour savoir en quels temps et lieux il faut semer et cultiver toutes choses qui servent en amitié et en amour, Paris, Sercy, 1657.

Almanach d'amour, pour l'an de grâce 1668 par le Grand Ovide, cyprois spéculateur des Éphémérides amoureuses. Aux remarques duquel se verront choses merveilleuses qui arriveront cette année. Dédié à Cupidon, à Chypre, chez Catelle, rue du Paradis à l'enseigne du coeur navré, 1668.

Almanach perpétuel d'amour selon les observations astronomiques de Cupidon. Diligemment supputé et réduit au Méridien du cour par Joly Passionné, professeur ès mathématiques d'amour, à l'île d'Adonis, par Fidèle Soupirant à la rue des Belles, à l'enseigne de Vénus, l'an 1681.

Almanach galant dédié au beau sexe pour l'année 1741, Paris, Gueffier, 1741.

Almanach des plaisirs, contenant les curiosités de Paris et de ses environs, et qui indique le temps des divertissements de la cour et de la ville. Avec des vaudevilles en pot-pourri, sur plusieurs danses de caractères les plus en usage. Dédié aux dames par M. C.**, Paris, Valleyre, 1757.

Almanach mignon, badin et divertissant dédié à tout le monde, Paris, Valleyre, 1762.

L'Astronomie galante. Étrennes au beau sexe, contenant une explication allégorique des planètes, des signes $d u$ zodiaque et des saisons en chansons, Paris, Cailleau, 1782.

Le Gaillard de bonne humeur, ou les plus courtes folies sont les meilleures, Amsterdam et Paris, s. n., 1786.

\section{Almanachs poissards et pornographiques}

Almanach de Priape, s. 1., 1741.

Almanach poissard, ou Étrennes grossières et inutiles, mais plaisantes et agréables, contenant plusieurs chansons grivoises des ports et des halles, avec des vaudevilles nouveaux sur des airs communs. Orné d'un bouquet poissard, à la Rapée, Pierrot Girous, avec permission des bateliers, 1757.

Almanach polisson ou Étrennes bouffonnes et poissardes, à la Courtille, chez Boniface Crouton, un des forts de la Halle, avec permission des blanchisseuses, 1759.

Étrennes poissardes et polissonnes, contenant plusieurs scènes facétieuses et gaillardes, avec des énigmes. Tant en chansons grivoises qu'en style poissard, aux porcherons, de l'impression de Marie Bonbec, la poule à ma tante, Paris, Gueffier puis Cailleau, 1763-1776.

Étrennes aux amateurs de Vénus, à Paphos ou Cythère, s. n., 1787.

Étrennes à Priape, ou recueil de chansons foutro-critico-énergico-lubriques dédiées à tous les crasseux disciples de Saint-François, par un bande-à-l'aise de la rue Tire-Boudin, Paris, l'an II de la liberté. 
Sylvain Maréchal, Almanach des honnêtes femmes pour l'année 1790, avec une gravure satirique originale du temps de la duchesse de Polignac, Bruxelles, Imprimerie de la Société Joyeuse, 1863.

Almanach des adresses des demoiselles de Paris, de tout genre et de toutes classes, ou Calendrier du plaisir contenant leurs noms, demeures, âges, portraits, caractères, talents et le prix de leurs charmes, enrichi de notes curieuses et anecdotes intéressantes, Paphos, de l'imprimerie de l'Amour, 1791 et Paris, Michel Héron, 1980.

Mes Priapines, Almanach nouveau dédié aux fouteurs de tout âge, de tout rang, et de tout sexe, 1791.

Étrennes aux fouteurs ou le calendrier des trois sexes, à Sodome et à Cythère et se trouvent plus qu'ailleurs dans la poche de ceux qui le condamnent, s. l., 1793.

\section{Almanachs bijoux}

Le Bijou mignon des dames, Paris, s. n., 1769.

Étrennes galantes, chez Vallages, marchand bijoutier, vers 1778 .

L'Almanach couleur de rose, ou les plus jolies étrennes chantantes, à Amathonte, chez Flore, au

Temple des Grâces et à Paris chez Cailleau, 1791. 